\title{
Indications and Safety of the Zygomatic Osteotomy in Middle Cranial Fossa Surgery: A Retrospective Cohort Review
}

\author{
Elliot Neal ${ }^{1}$ Elliot Pressman ${ }^{1} \quad$ Alexia Athienitis $^{2} \quad$ Adam Turner $^{1}$ Shunchang Ma ${ }^{1,3}$ Gautam Rao ${ }^{1}$ \\ Christopher Primiani ${ }^{1}$ Pankaj Agarwalla ${ }^{1}$ Harry van Loveren ${ }^{1}$ Siviero Agazzi ${ }^{1}$
}

\footnotetext{
1 Department of Neurosurgery and Brain Repair, University of South Florida, Tampa, Florida, United States

2 Muma College of Business, University of South Florida, Tampa, Florida, United States

${ }^{3}$ Department of Neurosurgery, Beijing Tiantan Hospital, Capital Medical University, Beijing, People's Republic of China
}

\begin{abstract}
Address for correspondence Siviero Agazzi, MD, Department of Neurosurgery and Brain Repair, USF Health South Health Tampa Center, 2 Tampa General Circle, Tampa, FL 33606 Tampa, FL 33602, United States (e-mail: sagazzi@health.usf.edu).
\end{abstract}

J Neurol Surg B 2019;80:225-231.

\begin{abstract}
Background Zygomatic osteotomy, an adjunct to middle cranial fossa (MCF) surgical approaches, improves the superior-inferior angle of approach and minimizes temporal lobe retraction. However, a decision-making algorithm for selective use of the zygomatic osteotomy and the impact of the zygomatic osteotomy on surgical complications have not been well documented.

Objective We described an algorithm for deciding whether to use a zygomatic osteotomy in MCF surgery and evaluated complications associated with a zygomatic osteotomy.

Methods A retrospective review of MCF cases over 11 years at our academic tertiary referral center was conducted. Demographic variables, tumor characteristics, surgical details, and postoperative complications were extracted.

Results Of the 87 patients included, 15 (17\%) received a zygomatic osteotomy. Surgical trajectory oriented from anterior to posterior (A-P) was significantly correlated with the use of the zygomatic osteotomy. Among the cases approached from A-P, we found (receiver-operating characteristic curve) that the cut-off tumor size that predicted a zygomatic osteotomy was $30 \mathrm{~mm}$. Of the 87 cases included, 15 patients had a complication. The multivariate logistic regression model failed to reveal any significant correlation between complications and zygomatic osteotomies.

\section{Keywords}

- zygomatic osteotomy

- middle cranial fossa

- skull base

- surgical approaches

- surgical adjuncts

Conclusions We found that the most important factor determining the use of a zygomatic osteotomy was anticipated trajectory. A-P approaches were most highly correlated with zygomatic osteotomy. Within those cases, a lesion size cut-off of $30 \mathrm{~mm}$ was the secondary predicting factor of zygomatic osteotomy use. The odds of suffering a surgical complication were not significantly increased by use of zygomatic osteotomy.
\end{abstract}

received

March 8, 2018

accepted after revision

July 7, 2018

published online

August 16, 2018
(C) 2019 Georg Thieme Verlag KG

Stuttgart · New York
DOI https://doi.org/

10.1055/s-0038-1668519. ISSN 2193-6331. 


\section{Introduction}

Surgical access to lesions in the middle cranial fossa (MCF), petrous apex, and cavernous sinus is usually gained with a temporal or frontotemporal craniotomy to which modifications such as anterior clinoidectomy, anterior petrosectomy, and zygomatic osteotomy are added to improve exposure while minimizing brain retraction. ${ }^{1}$ While the indications and benefits of each one of those modifications have been well, ${ }^{2-4}$ there is little data in the literature to document the impact of each one of those additions to the overall complications profile of those surgeries. Our group has previously evaluated the complications rate associated with an anterior petrosectomy and found no specific morbidity due to the use of an anterior petrosectomy during skull base approaches. ${ }^{2}$ In this study, we evaluated the impact of a zygomatic osteotomy on the surgical complications associated with skull base approaches through the middle fossa.

The history of middle fossa surgery goes back to the late 19th century when Harvey Cushing first accessed the trigeminal ganglion. ${ }^{5}$ The zygomatic osteotomy, an adjunct to MCF surgical approaches, improves the superior-inferior angle of approach and minimizes temporal lobe retraction. ${ }^{4,6-8}$ Due to the increased surgical access and maneuverability, the zygomatic osteotomy has become a popular adjunct in MCF surgery. The zygomatic arch is disconnected in two places: anteriorly at the union of the zygomatic arch and the zygoma and posteriorly just before the temporal-mandibular joint, ${ }^{7}$ with the masseter remaining attached. With the zygomatic arch reflected downward, the surgeon is better able to maneuver along the middle fossa floor with a lower trajectory to more effectively access the surgical field (- Fig. 1).

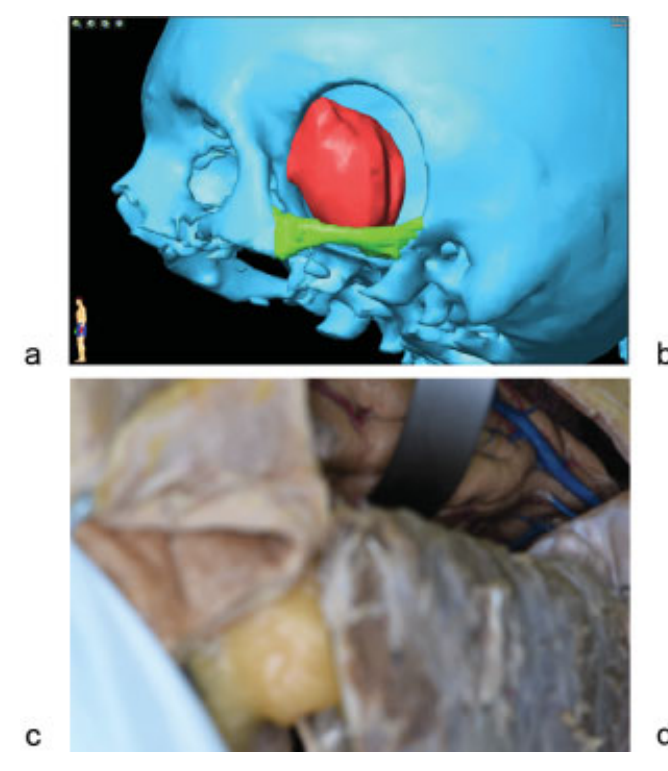

Over the past 11 years, the skull base practice at our tertiary academic referral center has evolved from the routine use of zygomatic osteotomies in approaches to the middle fossa to an attempt at a more limited use of such osteotomy to selected cases to minimize approach-related morbidity. The root of the zygomatic arch is situated on approximately the same coronal plane of foramen ovale, foramen spinosum, and foramen lacerum. We hypothesized that lesions located posterior to the root of the zygomatic arch would not benefit from a zygomatic osteotomy because they are not obstructed by the zygomatic arch, but that certain lesions lying anterior to the root may benefit. The zygomatic arch is rooted parallel to the floor of the middle fossa, but its arch component is situated adjacent to the anterior portion of the middle fossa floor. Therefore, we hypothesized that benefit conferred by removing the zygomatic arch would be maximized in cases where trajectory was oriented in a more anterolateral to posteromedial (A-P) direction (as opposed to straight lateral or posterolateral to anteromedial [P-A]). Also, larger tumors require a larger surgical window, any adjunct increasing that window would be beneficial for resection of large tumors. Furthermore, it is hypothesized that the use of a zygomatic osteotomy would result not only in a longer incision, but also would place titanium cranioplasty hardware directly underneath the wound, potentially causing longer healing time, more frequent infections, possibly extended hospital stay, and increased risk for associated complications. In our more conservative indications for zygomatic osteotomy, it was hypothesized that its use would be most beneficial in cases of large tumor resections and where an A-P trajectory was used. This study analyzes an 11-year long collection of patients who underwent surgery in the MCF to answer these questions.
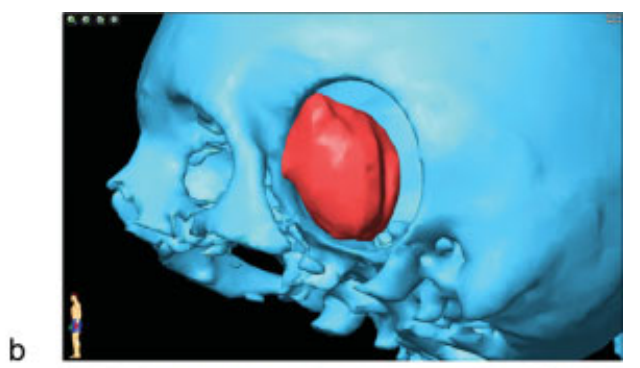

d

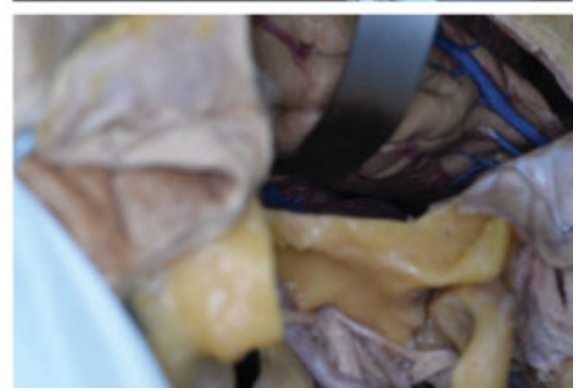

Fig. 1 A three-dimensional model of the surgical window is shown for a representative case where zygomatic osteotomy was used. (A) The skull (blue) is shown with a virtual surgical window revealing a meningioma (red). The zygomatic arch (green) covers the insertion of the tumor in the middle fossa floor. (B) With zygomatic arch removed, the insertion of the tumor is visualized and more easily accessed surgically. (C) Both images C and $\mathbf{D}$ are focused on the same point on the surface of the temporal lobe. A cadaveric dissection shows a similar viewpoint into the middle cranial fossa. The zygomatic arch and the reflected temporalis muscle obstructs the surgeon's view when the trajectory is oriented from an anterolateral to posteromedial direction. (D) Shows the same view with the zygomatic arch removed and the temporalis muscle reflected further inferiorly out of the surgical field, which increases the surgical window and allows for visualization of the middle fossa floor. 


\section{Materials and Methods}

\section{Patient Selection}

Data collection was conducted under approval from our institutional review board (IRB). As this was a retrospective study, prior consent was not obtained for those patients included in the study. However, the study did not alter the course of treatment or subject the patients to any additional risk, and therefore the need for consent was waived by the IRB. All patients under direct care of the senior author who underwent surgery via the MCF between 2005 and 2016 at our academic tertiary referral center were identified. In addition, one patient was excluded because his pre-surgical mental and cognitive status was the main determinant for the surgical goals and trajectory rather than the tumor itself. Another single patient was excluded because the tumor was not resected; only the dura mater was biopsied for staging purposes. This retrospective chart review established age at surgery, sex, lesion greatest diameter (if applicable), and surgical complications occurring within 30 days of surgery, including cerebrospinal fluid (CSF) leak, stroke, surgical site infection (SSI), return to operating room, pulmonary embolism (PE), deep vein thrombosis (DVT), urinary tract infection (UTI), and death.

\section{Tumor Location Classification}

For tumors approached using an MCF approach, tumor location was categorized into seven general locations: internal acoustic meatus, Meckel's cave, middle fossa floor, petroclival/cavernous-clival, sellar-suprasellar, sphenoclinoid/ spheno-cavernous/cavernous, or sphenoid wing. These locations were further divided as either being in the anterior half or the posterior half of the MCF. The demarcation between anterior and posterior halves of the MCF was the coronal plane intersecting with the root of the zygoma. Tumors located in the internal acoustic meatus and in the middle fossa floor, which mostly consisted of CSF leak repairs, were categorized as "posterior half MCF," and all other cases were categorized as "anterior half MCF."

\section{Zygomatic Osteotomy and Lesion Size Determination}

The independent variable, the use of a zygomatic osteotomy, was established by reviewing the operative summary note and confirming with postoperative computed tomography (CT) imaging where possible. Maximum lesion diameter for tumors was measured on either T1 with gadolinium or T2 preoperative magnetic resonance imaging (MRI).

\section{Approach Trajectory}

The anticipated approach trajectory was codified into the following three categories: A-P, straight lateral, and P-A (-Fig. 2). For example, a middle fossa meningioma inserted on the medial sphenoid wing would be classified as "anterolateral to posteromedial" while a trigeminal schwannoma occupying Meckel's cave would be classified as straight lateral. A "posterolateral to anteromedial" approach would be reserved for sellar and suprasellar lesions with retroclival extension. ${ }^{9}$ Surgical approach trajectory was determined prior to extraction of the dependent variables to minimize bias.

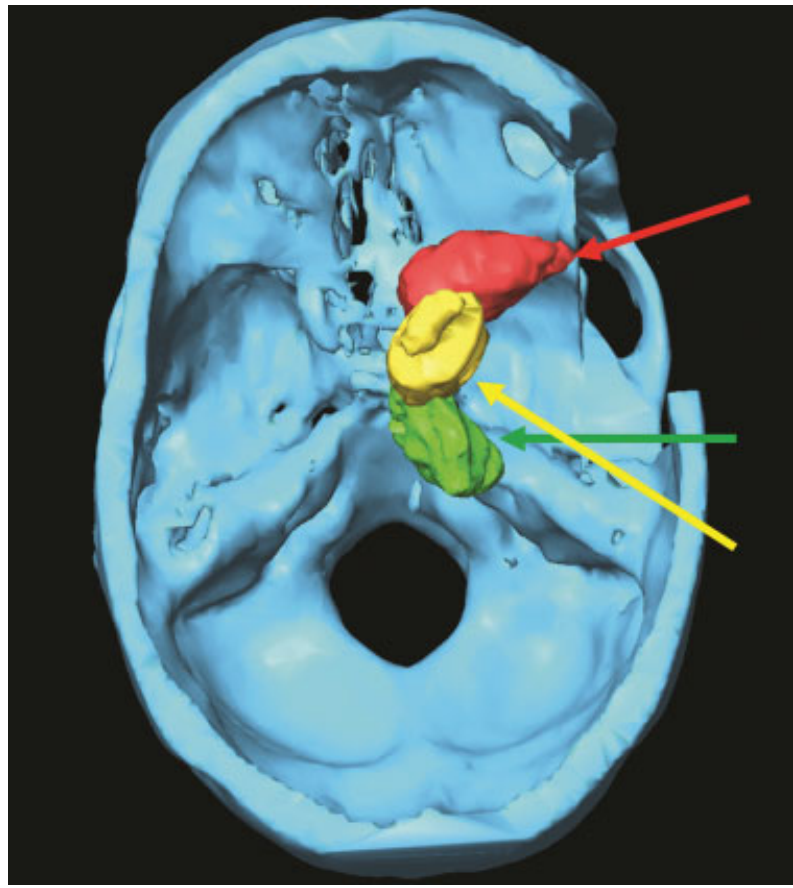

Fig. 2 The three surgical approach categories are shown with example cases from our series. The red trajectory shows the A-P trajectory. The green trajectory is a straight lateral example case, and the yellow trajectory shows a P-A trajectory example case. Only the A-P trajectory passes through the zygomatic arch, consistent with our finding that use of the zygomatic osteotomy was more useful in cases with an A-P approach trajectory. A-P, anterolateral to posteromedial; P-A, posterolateral to anteromedial.

\section{Statistical Analysis}

Data entry and statistical analysis were performed using IBM SPSS Statistics Version 24 (IBM Corp., Armonk, New York, United States) and SAS 9.4 (SAS Institute, Cary, NC, United States). A $p$-value $<0.05$ was considered statistically significant. A receiver-operating characteristics (ROC) curve was used for analysis of lesion diameter to determine the cutoff beyond which a zygomatic osteotomy was called for. Summary statistics were computed for all variables for patients with and without zygomatic osteotomy. Student's $t$-test was used to determine differences between the zygomatic osteotomy and non-zygomatic osteotomy groups for continuous variables, and the Mantel-Haenszel chi-square was used to compare categorical variables. Complication rate was calculated for both patients who did or did not have a zygomatic osteotomy, and significance was calculated after correcting for lesion diameter using a logistic regression model. We used logistic regression since the outcome was dichotomous (complication versus no complication), and that is the best model for our data given the nature of the variables. Logistic regression enables one to use regression models to predict the probability of a particular categorical response, in our case the complication, for a given set of independent variables (tumor size and zygomatic osteotomy). The logistic regression model uses the odds ratio, which represents the probability of an event of interest (a complication) compared with the probability of not having the event of interest. Subanalysis was done for tumor versus non-tumor patients to assess differences in trajectory approach, surgical complications, and the use of zygomatic osteotomy. 


\section{Results}

\section{Demographics}

A total of 87 patients were identified of which 32 were male and 55 were female. The age of the individuals ranged from 13 to 83 years, with a mean age of 54.0 years. For all cases conducted in the MCF, the tumors identified were primarily meningiomas (43 of 69 tumors) and also included 18 acoustic schwannomas, 1 trigeminal schwannoma, 5 craniopharyngiomas, and 2 epidermoid tumors. CSF leaks in the MCF floor comprised the remaining 18 of the 87 cases. Of the 87 cases identified, $15(17 \%)$ received a zygomatic osteotomy. There was no significant difference in patient gender or age between the zygomatic osteotomy or no zygomatic osteotomy groups (-Table $\mathbf{1}$ ).

\section{Use of the Zygomatic Osteotomy}

Our data clearly demonstrated that zygomatic osteotomy was categorically never used for tumors located in the posterior half of the MCF or for CSF leak repairs. We therefore eliminated all of those patients $(n=37)$ from any further analysis of the decision whether or not to use a zygomatic osteotomy. Of the 50 patients with pathologies in the anterior half of the MCF, a zygomatic osteotomy was used in 15 (30\%) cases. Lesion locations categories are shown in more detail in - Table 2 .

Table 1 Demographics

\begin{tabular}{|c|c|c|c|}
\hline & \multicolumn{2}{|l|}{ ZO } & \multirow[b]{2}{*}{$p$ Value } \\
\hline & $\begin{array}{l}\text { Yes } \\
(n=15)\end{array}$ & $\begin{array}{l}\text { No } \\
(n=72)\end{array}$ & \\
\hline Gender & & & 0.3718 \\
\hline Male $(n=32)$ & 4 & 28 & - \\
\hline Female $(n=55)$ & 11 & 44 & - \\
\hline Age at surgery & & & 0.3726 \\
\hline Mean (years \pm SD) & $56.9 \pm 13.0$ & $53.4 \pm 14.1$ & - \\
\hline Range (years) & $34-76$ & $13-83$ & - \\
\hline Diagnosis category & & & 0.0297 \\
\hline Tumor $(n=69)$ & 15 & 54 & - \\
\hline Other $(n=18)$ & 0 & 18 & - \\
\hline Etiology & & & 0.6992 \\
\hline CSF leak $(n=18)$ & 0 & 18 & - \\
\hline $\begin{array}{l}\text { Craniopharyngioma } \\
(n=5)\end{array}$ & 2 & 3 & - \\
\hline $\begin{array}{l}\text { Meningioma } \\
(n=43)\end{array}$ & 13 & 30 & - \\
\hline $\begin{array}{l}\text { Schwannoma } \\
(n=19)\end{array}$ & 0 & 19 & - \\
\hline $\begin{array}{l}\text { Other tumor } \\
(n=2)\end{array}$ & 0 & 2 & - \\
\hline $\begin{array}{l}\text { Length of stay } \\
\text { (days } \pm \text { SD) }\end{array}$ & $10.1 \pm 7.1$ & $5.3 \pm 6.5$ & 0.0497 \\
\hline
\end{tabular}

Abbreviations: CSF, cerebrospinal fluid; SD, standard deviation; ZO, zygomatic osteotomy.
Table 2 Tumor location

\begin{tabular}{|l|l|l|l|}
\hline & \multicolumn{2}{|l|}{ Z.O. } & \\
\hline & $\begin{array}{l}\text { Yes } \\
(\boldsymbol{n}=\mathbf{1 5})\end{array}$ & $\begin{array}{l}\text { No } \\
(\boldsymbol{n}=\mathbf{7 2})\end{array}$ & $p$ value \\
\hline Lesion location & & & $<0.0001$ \\
\hline $\begin{array}{l}\text { Posterior half of MCF } \\
(n=37)\end{array}$ & 0 & 37 & - \\
\hline $\begin{array}{l}\text { Internal acoustic } \\
\text { meatus ( } n=18)\end{array}$ & 0 & 18 & \\
\hline $\begin{array}{l}\text { Middle fossa floor } \\
(n=19)\end{array}$ & 0 & 19 & - \\
\hline $\begin{array}{l}\text { Anterior Half of MCF } \\
(n=50)\end{array}$ & 15 & 35 & \\
\hline $\begin{array}{l}\text { Meckel's cave } \\
(n=2)\end{array}$ & 0 & 2 & \\
\hline $\begin{array}{l}\text { Petroclival/ } \\
\text { cavernous-clival } \\
(n=6)\end{array}$ & 2 & 4 & \\
\hline $\begin{array}{l}\text { Sellar-suprasellar } \\
(n=5)\end{array}$ & 2 & 3 & \\
\hline $\begin{array}{l}\text { Sphenoclinoid/ } \\
\text { spheno-cavernous/ } \\
\text { cavernous ( } n=24)\end{array}$ & 7 & 17 & \\
\hline $\begin{array}{l}\text { Sphenoid wing } \\
(n=13)\end{array}$ & 4 & 9 & \\
\hline
\end{tabular}

Abbreviations: MCF, middle cranial fossa; ZO, zygomatic osteotomy.

The next step in deciding when to use a zygomatic osteotomy was surgical trajectory. Ninety percent were conducted using an A-P trajectory, 8.0\% straight lateral, and 2.0\% P-A. Zygomatic osteotomy was used in 33\% cases utilizing an A-P trajectory and for none of the cases using straight lateral or P-A approaches (-Table $\mathbf{3}$ ).

The final factor used to decide the use of the zygomatic osteotomy was the maximum lesion diameter. In cases where zygomatic osteotomy was used, the average tumor diameter was significantly $(p=0.0003)$ larger than tumors resected without zygomatic osteotomy ( - Fig. 3). An ROC. curve was created to estimate the tumor size threshold that best predicted use of a zygomatic osteotomy. At a lesion diameter cutoff of $30 \mathrm{~mm}$, zygomatic osteotomy was predicted with a specificity 0.846 and sensitivity of 0.769 ( - Fig. 4). The odds of using a zygomatic osteotomy in lesions $\geq 30 \mathrm{~mm}$ was greater by a factor of 5.8 (odds ratio) than for lesions $<30 \mathrm{~mm}$.

Table 3 Surgical trajectory direction (anterior half MCF subgroup)

\begin{tabular}{|c|l|l|}
\hline & ZO \\
\hline & $\begin{array}{l}\text { Yes } \\
(n=15)\end{array}$ & $\begin{array}{l}\text { No } \\
(n=35)\end{array}$ \\
\hline Anticipated trajectory & 15 & 30 \\
\hline Anterior to posterior $(n=45)$ & 15 & 4 \\
\hline Straight lateral $(n=4)$ & 0 & 1 \\
\hline Posterior to anterior $(n=1)$ & 0 &
\end{tabular}

Abbreviation: ZO, zygomatic osteotomy. 


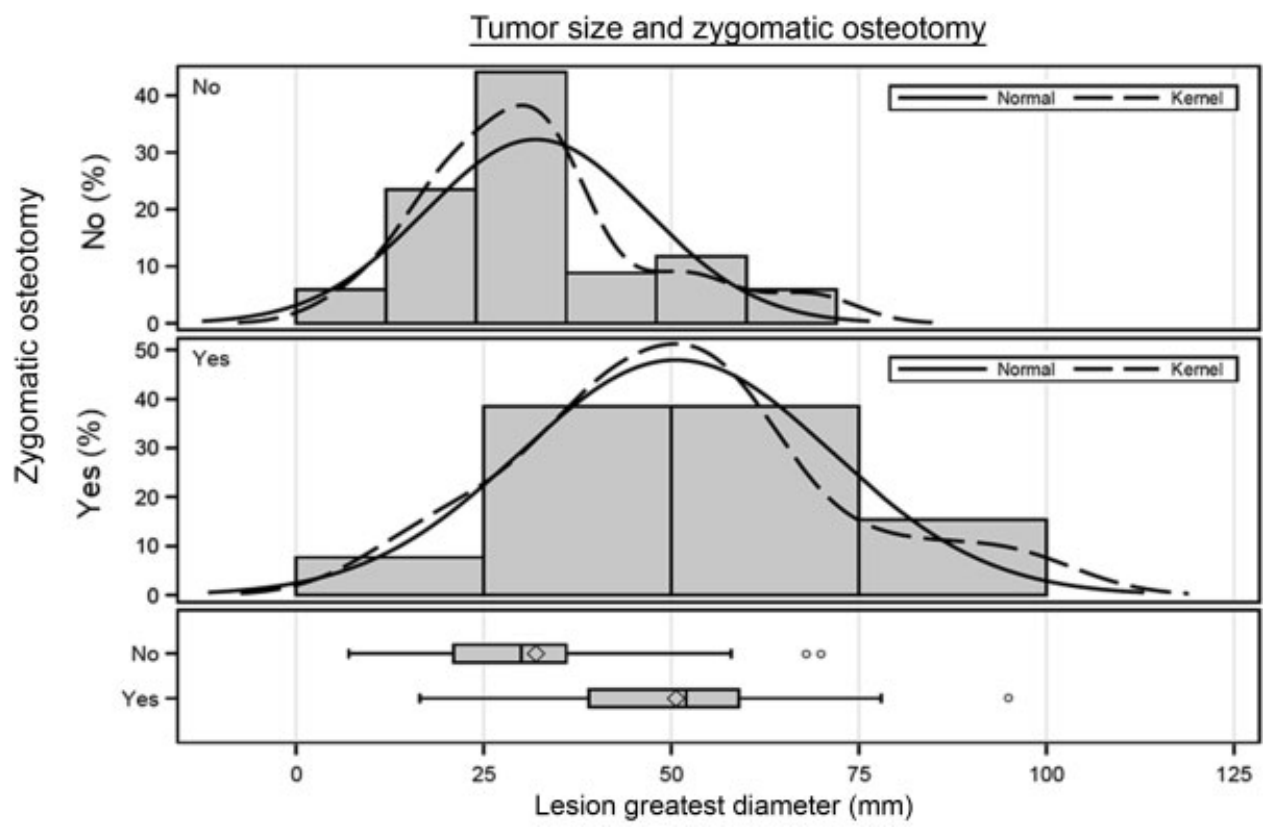

Fig. 3 For tumors in the anterior half of the middle cranial fossa, zygomatic osteotomy was used in cases to resect significantly $(p=0.0003)$ larger tumors than in cases that did not include zygomatic osteotomy $(50.7 \pm 20.8$ versus $28.7 \pm 16.3 \mathrm{~mm})$. Here, a histogram shows the distribution of lesion greatest diameter $(\mathrm{mm})$ in cases using zygomatic osteotomy (ZO = Yes) compared with cases where zygomatic osteotomy was not used. Overlaid on the histogram are two lines indicating an estimated normal distribution model (solid line) and Gaussian kernel (dashed line). A box and whisker plot shows the same data in a different format.

\section{Complications and Zygomatic Osteotomy}

The rate of surgical complications was considered the most important outcome measured in this study. For this analysis, we included all ( $n=87$ ) cases conducted in the MCF regardless of

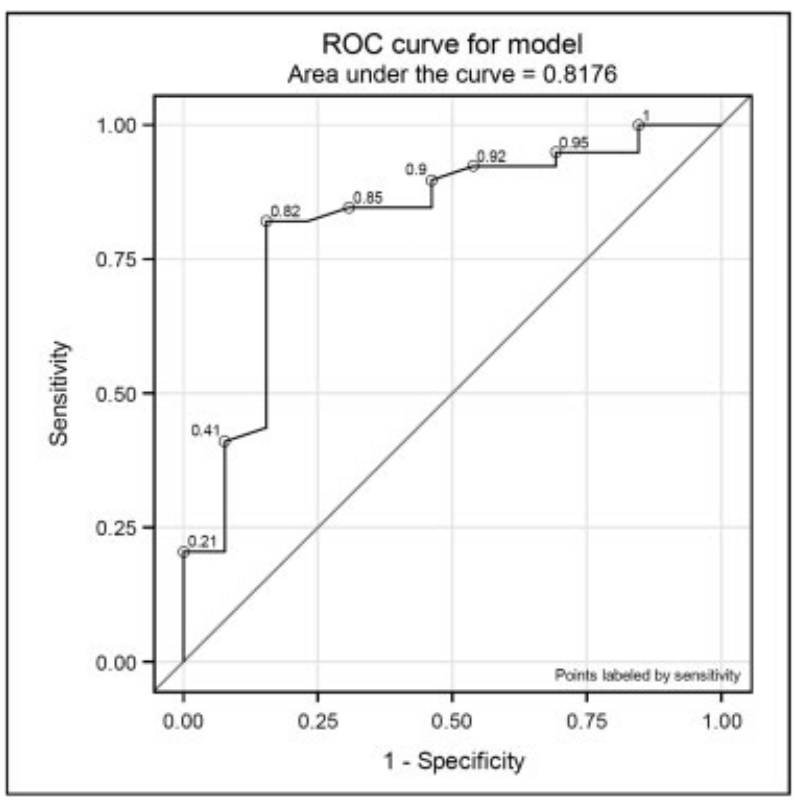

Fig. 4 For tumors in the anterior half of the middle cranial fossa, an estimated cutoff of $30 \mathrm{~mm}$ predicts use of zygomatic osteotomy with a specificity 0.846 and sensitivity of 0.769 . The cutoff was estimated by creating an ROC plot and selecting the lesion greatest diameter measurement that maximized sensitivity and specificity. ROC, receiver-operating characteristic. location or pathology. Of the 72 patients in this group that did not have a zygomatic osteotomy, 9 had a complication. Of the 15 patients who had a zygomatic osteotomy, 6 had a complication. Of the total cohort of patients who suffered a complication, six patients had a complication classified as "surgical" (SSI, CSF leak, stroke, additional surgery $<30$ days), and nine were classified as having a "systemic" complication (PE, DVT, UTI, and death). Reasons for additional surgeries within 30 days were as follows: CSF leak repair $(n=3)$, SSI $(n=1)$, hardware repositioning $(n=1)$, epidural hematoma $(n=2)$, and placement of ventriculoperitoneal shunt $(n=1)$. Detailed information about the surgical complications is contained within -Supplemental Table S1 (online only).

Univariate analysis showed that tumor diameter and use of zygomatic osteotomy both correlated highly with surgical complication rate. For this reason, analysis of the possible correlation between zygomatic osteotomy and surgical complication had to account for tumor size as a confounding variable. Multivariate logistic regression was used to correct for tumor diameter while measuring the effect of zygomatic osteotomy on surgical complications. The result showed that the use of a zygomatic osteotomy was not significantly predictive of a surgical complication $(p=0.67)$. Information about the multivariate logistic regression model is included in - Supplemental Table S2a-c (online only).

\section{Degree of Resection}

It was found that the degree of tumor resection was not significantly correlated to use of the zygomatic osteotomy with respect to both the surgeon's operative note $(p=0.46)$ and the radiologist's reading note $(p=0.77)$ ( - Table 4$)$. 
Table 4 Surgical outcomes

\begin{tabular}{|c|l|l|l|}
\hline Tumors only & \multicolumn{2}{|l|}{ Z.O. } & \\
\hline & $\begin{array}{l}\text { Yes } \\
(\boldsymbol{n}=\mathbf{1 5})\end{array}$ & $\begin{array}{l}\text { No } \\
(\boldsymbol{n}=\mathbf{3 7})\end{array}$ & - value \\
\hline $\begin{array}{l}\text { Degree of resection } \\
\text { (operative) }\end{array}$ & & 17 & 0.4583 \\
\hline Complete $(n=20)$ & 3 & 19 & - \\
\hline $\begin{array}{c}\text { Near complete/ } \\
\text { subtotal }(n=25)\end{array}$ & 6 & 1 & - \\
\hline Missing $(n=7)$ & 6 & 0.7695 \\
\hline $\begin{array}{l}\text { Degree of resection } \\
\text { (radiology) }\end{array}$ & & 17 & - \\
\hline Complete $(n=21)$ & 4 & 17 & - \\
\hline $\begin{array}{l}\text { Near complete/ } \\
\text { subtotal }(n=22)\end{array}$ & 5 & 3 & \\
\hline Missing $(n=9)$ & 6 & & \\
\hline
\end{tabular}

Abbreviation: ZO, zygomatic osteotomy.

\section{Discussion}

This study demonstrated our decision-making process for when to use a zygomatic osteotomy in MCF surgery. Lesions located in the posterior half of the MCF never required use of a zygomatic osteotomy regardless of surgical approach trajectory or tumor size. However, for lesions in the anterior half of the MCF, the most important determinant in the decision to remove a patient's zygomatic arch was the anticipated trajectory. Specifically, an A-P angle of approach was the most highly correlated with removal of the zygomatic arch. For patients with tumors, the size of tumor also influenced the decision of whether to perform a zygomatic osteotomy. Within the category of patients operated on with an anterolateral to posterior approach, the size cutoff of $30 \mathrm{~mm}$ was a significant determinant in the decision of whether to perform a zygomatic osteotomy. Our results relating to surgical complications indicated that after adjusting for tumor size, the odds of suffering a surgical complication were not significantly different between the groups of patients who underwent zygomatic osteotomy versus those who did not. Additionally, in nontumor MCF surgery patients, use of a zygomatic osteotomy was not associated with an increased risk of surgical complication.

In theory, a zygomatic osteotomy is done to increase the operative window, facilitate multidirectional access, and decrease temporal lobe retraction. ${ }^{8}$ The root of the zygomatic arch posteriorly is flush with the floor of the middle fossa and intersects a coronal plane at the approximate midpoint of the MCF. In cases where the tumor is located posterior to the root of the zygomatic arch, as is the case with acoustic schwannomas resected via an MCF approach, the zygomatic arch does not obstruct the surgical trajectory, and it is not necessary to remove it during surgery to improve access. However, as the zygomatic arch moves anteriorly it becomes thicker, especially in the superior-inferior dimension and blocks more of the middle fossa floor. As such, by removing the zygomatic arch when the angle of approach is
A-P, the operative window can be effectively enlarged. This corresponds to our findings that removal of the zygomatic arch was more common while approaching the surgical window in an A-P direction. However, there were cases in our series of patients with tumors that were resected with an A-P trajectory that did not have a zygomatic osteotomy, and the next decisive factor for those patients was maximum tumor diameter. An estimated tumor cutoff was determined statistically using an ROC curve that reflected this decisionmaking process; tumors $30 \mathrm{~mm}$ or larger in diameter were 5.8 times more likely to require use of a zygomatic osteotomy. Our results show that there exists a stepwise algorithm involved in the decision to perform a zygomatic osteotomy, incorporating lesion location, anticipated trajectory, and tumor size. So, is there a role for zygomatic osteotomy in small tumors? Definitively yes, in selected cases. Indeed, despite what our numbers show, each case should be considered individually, and the need for zygomatic osteotomy should be determined based on the goal to minimize temporal lobe retraction. A small tumor seated deep in the middle fossa floor might benefit from a zygomatic osteotomy, while a very large tumor displacing the temporal lobe posteriorly might be directly accessible after a simple pterional craniotomy.

Since the first description of the zygomatic osteotomy in the 1980s, skull base neurosurgeons have performed the procedure largely without studies supporting its safety. In 1995, Honeybul et al studied a cohort of patients and found a lack of increased morbidity associated with patients who underwent a zygomatic osteotomy. ${ }^{10}$ The results of our data support this. However, the more general topic of safety of the procedure has remained undiscussed. The efficacy of the zygomatic osteotomy on the other hand has been studied. The results are divided; some studies posit that with the additional maneuverability conferred by the zygomatic osteotomy more lesions can be reached safely, whereas others suggest that the operative window is relatively unimproved except for extensive lesions or those reaching into the infratemporal fossa. ${ }^{11-15}$ The majority of these studies focused on lesion location. However, in our study the lesion location was largely the same, the medial part of the MCF. Even when we categorized lesion location into distinct regions of the middle fossa, it was not found to be significantly predictive of zygomatic osteotomy use. This allowed us to focus on the anticipated trajectory-something previously unstudied. In addition, degree of tumor resection was presented for the sake of transparency and completeness, but we acknowledge the complex nature of resection degree as an outcome measure and therefore did not include it as a measure of efficacy. ${ }^{16}$

Our study had several limitations; it was a retrospective cohort study and had a modest sample size. A sample size of 15 is small, but that is the data that were available at that time. We could improve this model by conducting a prospective study, but that was not feasible at that time. As such, we were unable to analyze every factor that could exonerate the trans-zygomatic approach in surgical complications, for example the role of a zygomatic osteotomy in postoperative 
temporomandibular joint pain. As well, due to electronic medical record changes over the past 10 years, some data and follow-up information are unavailable, further limiting our sample size. However, it should be noted that the cases are all consecutive cases seen over an 11-year period; thus, the list of patients included in our study is still exhaustive.

\section{Conclusion}

Use of the zygomatic osteotomy is considered an option in certain surgical approaches to the MCF. For lesions located in the anterior half of the MCF, when the anticipated surgical trajectory is directed in the anterolateral-to-posteromedial direction, removal of the zygomatic arch offers the most advantage as compared with alternate trajectories because of the anatomical relationship between the zygomatic arch and the middle fossa floor. For larger tumors, with a size cut-off of 30-mm maximum diameter, zygomatic osteotomy was preferentially used because of the increased exposure of the surgical window needed to resect these larger tumors. Finally, use of zygomatic osteotomy did not increase the risk of surgery as measured by incidence of surgical complications. The zygomatic osteotomy is an important adjunct to middle fossa surgery and should be used selectively in cases only where it is appropriate.

\section{Note}

Previous Presentations: This study has been presented in part at the North American Skull Base Society $28^{\text {th }}$ annual meeting, February 14-15, 2018, Coronado, CA, United States.

\section{Funding \\ Not applicable.}

Financial Support

Not applicable.

Conflicts of Interest

None.

\section{References}

1 Chotai S, Kshettry VR, Petrak A, Ammirati M. Lateral transzygomatic middle fossa approach and its extensions: surgical technique and 3D anatomy. Clin Neurol Neurosurg 2015;130:33-41
2 Van Gompel JJ, Alikhani P, Youssef AS, Loveren HR, Boyev KP, Agazzi S. Anterior petrosectomy: consecutive series of 46 patients with attention to approach-related complications. J Neurol Surg B Skull Base 2015;76(05):379-384

3 Dolenc VV. A combined epi- and subdural direct approach to carotid-ophthalmic artery aneurysms. J Neurosurg 1985;62(05): 667-672

4 al-Mefty O, Anand VK. Zygomatic approach to skull-base lesions. J Neurosurg 1990;73(05):668-673

5 Cushing H. Landmark article April 28, 1900: a method of total extirpation of the Gasserian ganglion for trigeminal neuralgia. By a route through the temporal fossa and beneath the middle meningeal artery. By Harvey Cushing. JAMA 1983;250(04):519-528

6 Ammirati M, Ma J, Becker D, Black K, Cheatham M, Bloch J. Transzygomatic approach to the tentorial incisura: surgical anatomy. Skull Base Surg 1992;2(03):161-166

7 Campero A, Campero AA, Socolovsky M, et al. The transzygomatic approach. J Clin Neurosci 2010;17(11):1428-1433

8 Melamed I, Tubbs RS, Payner TD, Cohen-Gadol AA. Trans-zygomatic middle cranial fossa approach to access lesions around the cavernous sinus and anterior parahippocampus: a minimally invasive skull base approach. Acta Neurochir (Wien) 2009;151 (08):977-982, discussion 982

9 Wong RH, De Los Reyes K, Alikhani P, et al. The subtemporal approach to retroinfundibular craniopharyngiomas: a new look at an old approach. Oper Neurosurg (Hagerstown) 2015;11(04):495-503

10 Honeybul S, Neil-Dwyer G, Lang DA, Evans BT, Lees PD. The transzygomatic approach: a long-term clinical review. Acta Neurochir (Wien) 1995;136(3-4):111-116

11 Uttley D, Archer DJ, Marsh HT, Bell BA. Improved access to lesions of the central skull base by mobilization of the zygoma: experience with 54 cases. Neurosurgery 1991;28(01):99-103, discussion 103-104

12 Dayoub H, Schueler WB, Shakir H, Kimmell KT, Sincoff EH. The relationship between the zygomatic arch and the floor of the middle cranial fossa: a radiographic study. Neurosurgery 2010;66 (6, Suppl Operative)363-369

13 Ercan S, Scerrati A, Wu P, Zhang J, Ammirati M. Is less always better? Keyhole and standard subtemporal approaches: evaluation of temporal lobe retraction and surgical volume with and without zygomatic osteotomy in a cadaveric model. J Neurosurg 2017;127(01):157-164

14 Schwartz MS, Anderson GJ, Horgan MA, Kellogg JX, McMenomey SO, Delashaw JB Jr. Quantification of increased exposure resulting from orbital rim and orbitozygomatic osteotomy via the frontotemporal transsylvian approach. J Neurosurg 1999;91(06):1020-1026

15 Honeybul S, Neil-Dwyer G, Evans BT, Lang DA. The transzygomatic approach: an anatomical study. Br J Oral Maxillofac Surg 1997;35 (05):334-340

16 Ivan ME, Cheng JS, Kaur G, et al. Association of morbidity with extent of resection and cavernous sinus invasion in sphenoid wing meningiomas. J Neurol Surg B Skull Base 2012;73(01):76-83 